\title{
Biomarkers of Cardiac Stress and Cytokine Release Syndrome in COVID-19: A Review
}

\author{
Jonathan S. Gordon ${ }^{1} \cdot$ Mark H. Drazner ${ }^{1}$ (D) \\ Accepted: 2 February 2021 / Published online: 5 March 2021 \\ (C) The Author(s), under exclusive licence to Springer Science+Business Media, LLC part of Springer Nature 2021
}

\begin{abstract}
Purpose of Review The severe acute respiratory syndrome coronavirus 2 (SARS-CoV-2) resulted in the coronavirus 2019 (COVID-19) global pandemic. While primarily a respiratory virus, SARS-CoV-2 can cause myocardial injury. The pattern of injury, referred to as acute COVID-19 cardiovascular syndrome (ACovCS), is defined by cardiac troponin leak in the absence of obstructive coronary artery disease. Although the etiology of the injury is unknown, many speculate that a cytokine release syndrome (CRS) may be an important factor. We aim to review recent data concerning markers of cardiac injury in ACovCS and its relation to the CRS.

Recent Findings Cardiac injury was common in patients hospitalized for COVID-19, with both cardiac troponin and B-type natriuretic peptide (BNP) being elevated in this population. Biomarkers were correlated with illness severity and increased mortality. Cytokines such as IL-6 were more often elevated in patients with ACovCS. Myocarditis evident on cardiac MR following COVID-19 may be associated with cardiac troponin levels. The impact of dexamethasone and remdesivir, two therapies shown to have clinical benefit in COVID-19, on myocardial injury is unknown.

Summary Biomarkers of cardiac stress and injury in COVID-19 may be used to stratify risk in the future. Currently, there is no evidence that inhibition of cytokine release will reduce myocardial injury in patients with COVID-19.
\end{abstract}

Keywords COVID-19 · coronavirus · cardiac biomarkers · troponin · BNP · cytokine release syndrome

\section{Introduction}

Coronavirus disease 2019 (COVID-19) is a global pandemic caused by the severe acute respiratory syndrome coronavirus 2 (SARS-CoV-2) [1]. The clinical syndrome was first described in December 2019 as a cluster of individuals who presented with "viral pneumonia" [1] in Wuhan, China. The first case in the USA was detected shortly thereafter in January 2020 [2]. The virus has since spread globally and infected

This article is part of the Topical Collection on Biomarkers of Heart Failure

Mark H. Drazner

Mark.drazner@UTsouthwestern.edu

Jonathan S. Gordon

Jonathan.gordon@UTsouthwestern.edu

1 Department of Internal Medicine and Division of Cardiology, University of Texas Southwestern Medical Center, 5323 Harry Hines Blvd, Dallas, TX 75390-9254, USA more than 50 million individuals, resulting in over 1.3 million deaths [3].

In addition to the respiratory and systemic manifestations of COVID-19, an acute cardiovascular syndrome (ACovCS) has been described [4]. ACovCS includes a myocarditis-like syndrome, with cardiac troponin leak in the absence of obstructive coronary artery disease $[4,5]$. Myocardial injury in the setting of COVID-19 may lead to arrhythmias and heart failure with reduced ejection fraction, sometimes evolving to overt shock [4]. However, the exact etiology of myocardial injury has not been elucidated. To date, there have been several proposed mechanisms, including direct viral infection of cardiac cells, microvascular dysfunction, and cytokine release syndrome (CRS)-mediated myocardial damage [4, 6]. Much of the understanding of ACovCS has evolved from measurements of biomarkers associated with myocardial stress and injury. This article aims to review current data on the biomarkers associated with ACovCS and cytokine release syndrome in COVID-19, with a focus on their diagnostic and prognostic utility as well as their role in guiding treatment. 


\section{Cardiac Troponin}

ACovCS with elevated cardiac troponins is common in SARS-CoV-2 [4], although estimates on its prevalence vary widely. A single-center retrospective analysis from Wuhan, including 273 patients admitted with COVID-19, found ultra-TnI was elevated $(>0.04 \mathrm{ng} / \mathrm{mL}$ ) at admission in just $9.9 \%(27 / 273)$ of patients [7]. In contrast, another retrospective single-center study found that nearly $20 \%(82 / 416)$ of patients hospitalized with COVID-19 had elevated $(\geq 0.04$ $\mathrm{ng} / \mathrm{mL}$ ) high sensitivity cardiac troponin I (hs-TnI) [8]. Other studies have found even a higher prevalence of elevated cardiac troponin levels. In 397 patients at a center converted for regional, tertiary care of COVID-19 patients in Italy, 32\% $(130 / 397)$ were found to have elevated hs-TnI ( $\geq 19.6 \mathrm{ng} / \mathrm{L}$ ) on admission [9]. Another study from Wuhan found 27.8\% with myocardial injury as assessed by cardiac troponin $\mathrm{T}$ ( $>$ 99th percentile upper reference range) [10••]. In this study, hospitalized patients with versus without preexisting cardiovascular disease were more likely to have elevated cardiac troponins $(54.5 \%$ vs. $13.2 \%$ ) [10••]. Furthermore, the troponin levels were dynamic in some patients. TnT increased significantly from baseline in the period before death in those that ultimately died but not in those that survived [10••].

Myocardial injury as assessed by elevated cardiac troponin was associated with worse outcomes in patients with COVID19. In the single-center cohort from Wuhan with 416 patients hospitalized with COVID-19, elevated hs-TnI was associated with increased utilization of both non-invasive and invasive mechanical ventilation, acute respiratory distress syndrome (ARDS), and increased mortality rate $(51.2 \%$ vs. $4.5 \% ; P<$ 0.001 ) [8]. Furthermore, mortality rate was directly proportional to the degree of hs-TnI elevation $(11.1 \%$ if between 0.006 and $0.04 \mathrm{ng} / \mathrm{mL} ; 36 \%$ between 0.04 and $0.78 \mathrm{ng} / \mathrm{m}$; $92.3 \%$ if $>0.78 \mathrm{ng} / \mathrm{mL}$ ) [8]. Another retrospective review from two hospitals in Wuhan, comprised of 150 hospitalized patients with COVID-19, found that cardiac troponins were elevated in patients that died compared to those that were ultimately discharged $(30.3 \mathrm{pg} / \mathrm{mL}$ vs. $3.5 \mathrm{pg} / \mathrm{mL}, p<0.001$, respectively) [11]. These findings were further corroborated in another single-center retrospective case series of hospitalized patients with COVID-19 in which $59.6 \%$ (31/52) with elevated TnT (> 99th percentile) compared to only $8.9 \%(12 / 135)$ with normal values died [10••]. Elevated TnT was also associated with malignant arrhythmias $(11.5 \%$ vs. $5.2 \%)$ and ARDS $(57.7 \%$ vs. $11.9 \%$ ) [10••]. A metanalysis of 341 patients at four centers in Wuhan found cardiac troponin I was more likely to be elevated in patients with severe COVID-19 disease, as defined by mechanical ventilation, ICU admissions, or death (mean difference $25.6 \mathrm{ng} / \mathrm{mL}$; 95\% CI 6.8 $44.5 \mathrm{ng} / \mathrm{mL}$ ) [12]. However, the studies included in this analysis had significant heterogeneity in reported cardiac troponin concentrations. A larger meta-analysis from centers predominantly in China, including 13 studies with 2389 patients, found that cardiac injury (hs-TnI $>99$ th percentile) was associated with higher mortality (RR 7.95; 95\% CI 5.12 12.34; $P<0.001)$ compared to those without cardiac injury [13••]. The composite endpoint of mechanical ventilation, ARDS, ICU admissions, or death was also associated with higher levels of hs-TnI (mean difference $=10.38 \mathrm{pg} / \mathrm{mL}$; 95\% CI 4.44-16.32; $P=0.002$ ) [13••]. These findings have largely been replicated by more recent data. For example, in one study from Italy consisting of 397 consecutive patients with COVID-19, patients with versus without elevated hs-TnI $(\geq 19.6 \mathrm{ng} / \mathrm{L})$ had increased mortality $(22.5 \%$ vs. $6.25 \%$; OR $4.35,95 \%$ CI $1.7-11$ ) [9].

\section{B-type Natriuretic peptides}

B-type natriuretic peptide (BNP) and N-terminal proBNP (NT-proBNP) are markers of myocardial stress [14]. BNP and NT-proBNP are elevated in critical illness and associated with adverse outcomes in non-cardiac disease processes such as sepsis $[15,16]$. It was therefore of interest to determine if natriuretic peptides were associated with cardiac injury and outcomes in patients with COVID-19.

NT-proBNP was found to be elevated in patients with COVID-19 who have myocardial injury. A study of 397 consecutive patients from a COVID-19 center in Italy reported that $37.5 \%$ (149/397) of patients with COVID-19 had elevated $\mathrm{BNP}(\geq 100 \mathrm{pg} / \mathrm{mL})$ on admission, of which 90 (23\% of overall cohort) had both elevated hs-TnI and BNP [9]. In a singlecenter analysis from Wuhan, NT-proBNP levels were elevated in those with versus without myocardial injury $(1689 \mathrm{pg} /$ $\mathrm{mL}$ vs. $139 \mathrm{pg} / \mathrm{mL}$, respectively, $P<0.001$ ) [8]. Furthermore, NT-proBNP was also significantly elevated in those with versus without elevated $\mathrm{TnT}$ ( $>$ 99th percentile) (817.4 vs. 141.4 $\mathrm{pg} / \mathrm{mL} ; P<0.001)$ in another single-center study $[10 \bullet$. Similar to TnT, NT-proBNP levels evolved during hospital admission in certain patients with COVID-19. For example, NT-proBNP levels increased significantly after admission in patients who subsequently died but not in those who survived the hospitalization [9]. There was also a positive correlation between TnT levels and NT-proBNP $(\beta=0.613 ; P<0.001)$ $[10 \bullet \cdot$.

NT-proBNP also is a marker of disease severity in patients with COVID-19 and has prognostic value. In the single-center study by Han et al. of 273 patients in Wuhan, 12.4\% (34/273) were found to have elevated NT-proBNP $(\geq 900 \mathrm{pg} / \mathrm{mL})$ at admission [7]. This same study found NT-proBNP was significantly increased in "severe" and "critical" cases of COVID-19 as compared to "mild" cases. Additionally, both NT-proBNP and cardiac troponin were elevated in those that later died compared to those that remained alive at follow-up $(\mathrm{P}<0.001)$ [7]. In a multicenter retrospective study of 9 
hospitals in Hubei province including 3219 patients with COVID-19, concentrations of BNP and/or NT-proBNP were elevated in $12.9 \%$ and associated with increased 28-day mortality (HR 5.11; 95\% CI 3.50-7.47; $P<0.001$ ) even after adjusting for age, sex, and coexisting conditions [17]. The combination of elevated cardiac troponin and NT-proBNP appeared to identify a particularly high-risk group in multivariable regression controlling for additional comorbidities and laboratory abnormalities (OR 3.24; 95\% CI 1.06-9.93, $P=$ 0.039 ) [9]. Finally, the utility of cardiac biomarkers to assess risk may be improved by incorporating different reference ranges. For example, in one study, lower cutoffs for cardiac biomarkers, e.g., cardiac troponin $49 \%$ ULN and (NT-pro) BNP 18.8\% ULN, improved predictive models [17].

\section{Cardiac Biomarkers and COVID-19-Associated Myocarditis on CMR}

Studies reporting the prevalence of myocarditis, as assessed by cardiac MR (CMR), in patients with COVID-19 are summarized in Table 1. The association between myocardial edema on CMR and cardiac injury, as measured by biomarkers, remains uncertain. In one study, 15\% (4/26) of college athletes with COVID-19 had CMR evidence of myocarditis despite a normal ECG, transthoracic echocardiogram, and cardiac troponin I levels [18]. Another study found 58\% (15/26) of COVID-19 patients with cardiac symptoms had abnormal CMR findings, including 54\% (14/26) with myocardial edema and $31 \%(8 / 26)$ with late gadolinium enhancement [19]. None of these patients had elevated hs-TnI at the time of imaging, and there was no statistically significant difference in the levels of NT-proBNP or IL- 6 between patients with and without CMR findings [19]. However, in another case series, high sensitivity cardiac troponins were elevated in all 10 patients with CMR evidence of myocardial edema, although 2 patients likely had Takotsubo cardiomyopathy [20]. A larger prospective study of 100 patients recently recovered from COVID-19 found $78 \%$ had abnormal cardiac MRI findings [21•]. In that study, hs-TnT concentrations were significantly correlated with both native T1 mapping $(r=0.35 ; P<0.001)$ and native T2 mapping $(r=0.22 ; P=0.03)$, while both NT-proBNP and CRP levels were not [21•]. The correlation of CMR T1 and T2 mapping with cardiac troponin levels persisted in multivariable models after controlling for confounders such as key comorbidities. A limitation of the above CMR studies was that the imaging test and biomarker concentrations were obtained weeks to months after the acute infection phase of COVID-19. There are no data from hospitalized patients, as CMRs were not performed on clinical grounds in the setting of critical COVID-19 illness. Further data are needed on this important subject.

\section{Cytokine Storm}

Cytokine storm has been implicated as a potential etiology of the myocardial injury that can be seen in some patients with COVID-19 [4, 6]. Cytokine storm syndrome is an acute hyperinflammatory response characterized by cytokine release and malignant activation of the inflammatory cascade [22-24]. While there is no clear consensus on what constitutes a cytokine storm, acute systemic inflammatory symptoms in the setting of circulating inflammatory cytokines and secondary organ dysfunction beyond a normal pathogenic response has recently been proposed as a unifying definition [24]. Perhaps in an analogous fashion, a cytokine response syndrome (CRS) occurs following chimeric antigen receptor $\mathrm{T}$ cell (CAR-T) therapy. In that setting, the degree of cardiac injury was associated with a higher grade of cytokine release syndrome [25].

Patients with COVID-19, especially more severe forms, have elevated levels of a number of cytokines including IL2, IL-2R, IL-6, IL-7, IL-10, IFN- $\gamma$, TNF- $\alpha$, VEGF, and MIP $1 \alpha$ and $1 \beta[23,26,27]$. The immune dysregulation and resulting cytokine syndrome may result in critical illness and multiorgan failure, including cardiac dysfunction [28]. In a prospective single-center study from Wuhan with 41 hospitalized COVID-19 patients, those admitted to the ICU had higher levels of IL-2, IL-7, IL-10, GCSF, and TNF- $\alpha$ [29]. Further, elevated levels of cytokines were associated with mortality in patients with COVID-10. In a retrospective study from two hospitals in Wuhan, those patients who succumbed to COVID-19 vs. those who survived had elevated IL-6 levels $(11.4 \mathrm{ng} / \mathrm{mL}$ vs. $6.8 \mathrm{ng} / \mathrm{mL} ; P<0.0001$, respectively) [11]. In another retrospective cohort study of patients with COVID-19 from two Wuhan centers, IL-6 was elevated both at admission and throughout the hospital course in those who did not survive COVID infection as compared to survivors [30].

Levels of inflammatory biomarkers have been shown to be associated with those of cardiac troponin in patients with COVID-19. Levels of procalcitonin (median 0.21 vs. 0.05 $\mathrm{mg} / \mathrm{dL} ; P<0.001$ ) and C-reactive protein (median 8.55 vs. $3.13 \mathrm{mg} / \mathrm{dL} ; P<0.001)$ were significantly higher in hospitalized COVID-19 patients with elevated TnT than those without elevated TnT [10••]. Furthermore, higher C-reactive protein levels were strongly correlated with increasing plasma TnT $(\beta=0.53 ; P<0.001)\left[10^{\bullet \bullet}\right]$ suggesting a link between inflammation and myocardial injury. These findings were corroborated in a retrospective single-center study of 416 patients in which cardiac injury was associated with elevated C-reactive protein (median 10.2 vs. $3.7 \mathrm{mg} / \mathrm{dL}$ ) and procalcitonin (median 0.27 vs. $0.06 \mathrm{ng} / \mathrm{mL}$ ) [8]. CRP may also serve as an early warning sign of cardiac injury as its elevation preceded the rise in cardiac troponin [16]. In contrast, IL-6 significantly elevated after the rise in cardiac troponin [16]. 


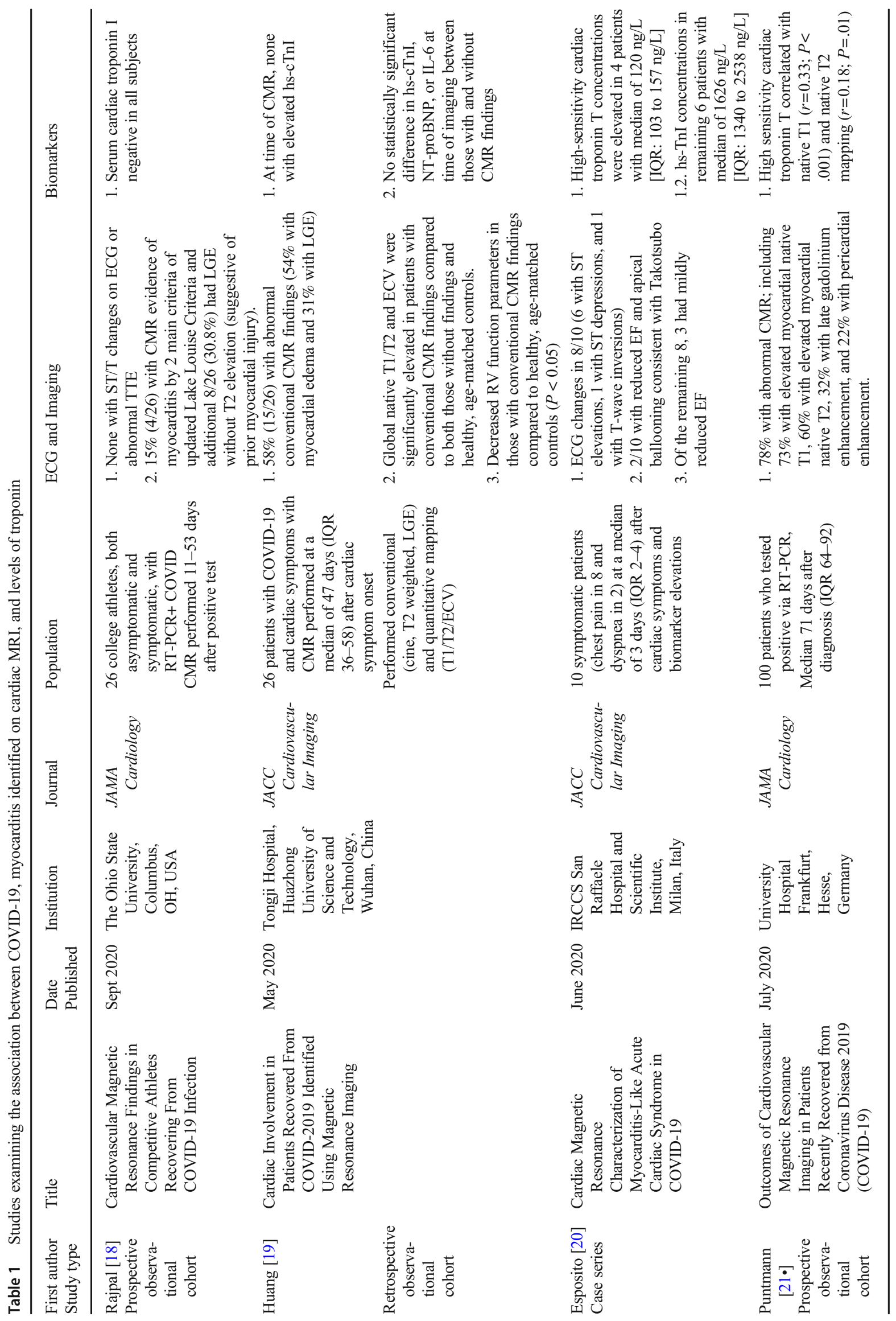


While therapeutics aimed directly at immune mediators of CRS exist, there is scant evidence of clinical benefit in patients with COVID-19 overall or for ACovCS specifically. Tocilizumab is a FDA-approved IL-6 inhibitor used to treat CRS related to CAR-T therapy [31]. While the initial data from retrospective cohorts in patients with COVID were promising [32], a prospective randomized controlled trial found no difference in rates of intubation or death, clinical worsening, and duration of supplemental oxygen use with tocilizumab compared to placebo [33]. The IL-1 inhibitor, anakinra, has also been explored as a treatment for COVID-19. While there are small retrospective studies suggesting benefit with anakinra therapy [34, 35], there are no prospective clinical trial data in patients with COVID-19 yet available. Some data suggested that early tocilizumab administration after CRS related to CAR-T therapy may reduce risk of cardiovascular injury [25]. However, trials of IL-6 and IL-1 inhibitors have not examined their influence on the prevalence and magnitude of cardiac injury in patients with COVID-19 and/or ACovCS.

\section{Impact of Therapies Effective in COVID-19 on Cardiac Injury}

Currently, dexamethasone [36] and remdesivir [37] are the only two treatments demonstrated in large randomized clinical trials to have benefit on clinical outcomes in patients with COVID-19. However, data on how these agents impact cardiac injury are limited. In the RECOVERY trial, dexamethasone vs. standard care reduced the rate of 28-day mortality in patients with COVID-19 who required oxygen therapy at randomization [36]. Data regarding the impact of dexamethasone on cardiac arrhythmias from the RECOVERY trial are being collected but have not yet been released. Remdesivir, a viral RNA polymerase inhibitor, was shown in the adaptive, randomized controlled ACTT-1 study to reduce median recovery time compared to placebo ( 10 vs. 15 days; rate ratio for recovery $1.29 ; 95 \%$ CI $1.12-1.49 ; P<0.001$ ) [37]. In that trial, the frequency of increased cardiac troponin, reported as an adverse event, for those who received remdesivir versus placebo was $0.2 \%$ vs. $1.0 \%$ [37].

\section{Conclusion and Future Directions}

It remains uncertain how best to incorporate measurement of biomarkers in patients with COVID-19. Earlier in the pandemic, routine testing of cardiac troponin in patients with COVID-19 was not advised unless an acute coronary syndrome was suspected [38]. However, some now recommend that serial measurements of troponin be performed in patients admitted for COVID-19 [39]. Testing all patients on admission may help risk stratify and enhance triage of patients, a task which is particularly important given the pandemic-related stress on the health care system limiting bed and ICU availability.

While new therapies are available for the treatment of COVID-19, few studies have examined whether they favorably impact cardiac injury and that is an area in need of further investigation. Although it appears that increased cardiac troponin levels in the setting of COVID-19 do not commonly result in ventricular dysfunction, cardiac troponin concentrations nevertheless were associated with increased mortality and other adverse outcomes. Given that the pandemic started less than a year ago, little is known about the long term cardiovascular prognostic implications of detectable cardiac troponin levels during an acute COVID-19 illness. Nevertheless, it is plausible that therapies targeted towards reducing myocardial injury have the potential to improve both short-term mortality and help mitigate any unknown long-term cardiac consequences of COVID-19 infection.

\section{Declaration}

Conflict of Interest Dr. Jonathan Gordon and Dr. Mark Drazner declare they have no conflict of interest.

Human and Animal Rights and Informed Consent This article does not contain any studies with human or animal subjects performed by any of the authors.

\section{References}

Papers of particular interest, published recently, have been highlighted as:

- Of importance

•- Of major importance

1. Pneumonia of unknown cause - China. World Heal. Organ. Website. https://www.who.int/csr/don/05-january-2020pneumonia-of-unkown-cause-china/en/. Published 1/5/2020. Accessed 11/19/2020

2. Holshue ML, DeBolt C, Lindquist S, Lofy KH, Wiesman J, Bruce $\mathrm{H}$, et al. First case of 2019 novel coronavirus in the United States. N Engl J Med. 2020;382:929-36.

3. COVID-19 Dashboard by the Center for Systems Science and Engineering (CSSE) at Johns Hopkins University (JHU). Johns Hopkins Univ. Coronavirus Resour. Cent. Website. https:// coronavirus.jhu.edu/map.html. Published 11/19/2020. Accessed 11/ $19 / 2020$.

4. Hendren NS, Drazner MH, Bozkurt B, Cooper LT Jr. Description and proposed management of the acute COVID-19 cardiovascular syndrome. Circulation. 2020;141(23):1903-14.

5. Hendren NS, Grodin JL, Drazner MH. Unique patterns of cardiovascular involvement in coronavirus disease-2019. J Card Fail. 2020;26(6):466-9.

6. Tersalvi G, Vicenzi M, Calabretta D, Biasco L, Pedrazzini G, Winterton D. Elevated troponin in patients with coronavirus disease 2019: possible mechanisms. J Card Fail. 2020;26(6):470-5.

7. Han H, Xie L, Liu R, Yang J, Liu F, Wu K, et al. Analysis of heart injury laboratory parameters in 273 COVID-19 patients in one hospital in Wuhan, China. J Med Virol. 2020;92:819-23. 
8. Shi S, Qin M, Shen B, Cai Y, Liu T, Yang F, et al. Association of cardiac injury with mortality in hospitalized patients with COVID19 in Wuhan, China. JAMA Cardiol. 2020;5:802-10.

9. Stefanini GG, Chiarito M, Ferrante G, Cannata F, Azzolini E, Viggiani G, et al. Early detection of elevated cardiac biomarkers to optimise risk stratification in patients with COVID-19. Heart. 2020;106:1512-8.

10.• Guo T, Fan Y, Chen M, Wu X, Zhang L, He T, et al. Cardiovascular implications of fatal outcomes of patients with coronavirus disease 2019 (COVID-19). JAMA Cardiol. 2020;5:811-8 This study shows that NT-proBNP was associated with elevated troponin levels in patients with COVID-19 and that these values increased over time in those that had worse outcomes.

11. Ruan Q, Yang K, Wang W, Jiang L, Song J. Clinical predictors of mortality due to COVID-19 based on an analysis of data of 150 patients from Wuhan, China. Intensive Care Med. 2020;46(5):846-8.

12. Lippi G, Lavie CJ, Sanchis-Gomar F. Cardiac troponin I in patients with coronavirus disease 2019 (COVID-19): Evidence from a metaanalysis. Prog Cardiovasc Dis. 2020;63(3):390-1.

13.• Santoso A, Pranata R, Wibowo A, Al-Farabi MJ, Huang I, Antariksa B. Cardiac injury is associated with mortality and critically ill pneumonia in COVID-19: A meta-analysis. Am J Emerg Med. 2020;:0735-6757(20):30280-1 This study is the largest analysis showing elevated troponins were associated with increased mortality in patients with COVID-19.

14. de Lemos JA, McGuire DK, Drazner MH. B-type natriuretic peptide in cardiovascular disease. Lancet. 2003;362:316-22.

15. Varpula M, Pulkki K, Karlsson S, Ruokonen E, Pettilä V. Predictive value of N-terminal pro-brain natriuretic peptide in severe sepsis and septic shock. Crit Care Med U S. 2007;35:1277-83.

16. Vallabhajosyula S, Wang Z, Murad MH, Vallabhajosyula S, Sundaragiri PR, Kashani K, et al. Natriuretic peptides to predict short-term mortality in patients with sepsis: a systematic review and meta-analysis. Mayo Clin Proceedings Innov Qual Outcomes. 2020;4:50-64.

17. Qin J-J, Cheng X, Zhou F, Lei F, Akolkar G, Cai J, et al. Redefining cardiac biomarkers in predicting mortality of inpatients with COVID-19. Hypertens (Dallas, Texas). 2020;76:1104-12.

18. Rajpal S, Tong MS, Borchers J, Zareba KM, Obarski TP, Simonetti $\mathrm{OP}$, et al. Cardiovascular magnetic resonance findings in competitive athletes recovering from COVID-19 infection. JAMA Cardiol. $2020 \mathrm{Sep} ; 11: \mathrm{e} 2-4016$.

19. Huang L, Zhao P, Tang D, Zhu T, Han R, Zhan C, et al. Cardiac involvement in patients recovered from COVID-2019 identified using magnetic resonance imaging. JACC Cardiovasc Imaging. 2020;13:2330-9.

20. Esposito A, Palmisano A, Natale L, Ligabue G, Peretto G, Lovato $\mathrm{L}$, et al. Cardiac magnetic resonance characterization of myocarditis-like acute cardiac syndrome in COVID-19. JACC Cardiovasc Imaging. 2020;13(11):2462-5.

21. Puntmann VO, Carerj ML, Wieters I, Fahim M, Arendt C, Hoffmann J, et al. Outcomes of cardiovascular magnetic resonance imaging in patients recently recovered from coronavirus disease 2019 (COVID-19). JAMA Cardiol. 2020;5(11):1265-73 This prospective study provides the best evidence of association between abnormal cardiac MR findings and elevated markers of caridac injury.

22. Mehta P, McAuley DF, Brown M, Sanchez E, Tattersall RS, Manson JJ. COVID-19: consider cytokine storm syndromes and immunosuppression. Lancet (London, England). 2020;395:1033-4.

23. Guo Y-R, Cao Q-D, Hong Z-S, Tan Y-Y, Chen S-D, Jin H-J, et al. The origin, transmission and clinical therapies on coronavirus disease 2019 (COVID-19) outbreak - an update on the status. Mil Med Res. 2020;7:11.
24. Fajgenbaum DC, June CH. Cytokine Storm. N Engl J Med. 2020;383:2255-73.

25. Alvi RM, Frigault MJ, Fradley MG, Jain MD, Mahmood SS, Awadalla M, et al. Cardiovascular events among adults treated with chimeric antigen receptor T-cells (CAR-T). J Am Coll Cardiol. 2019;74:3099-108.

26. Chen G, Wu D, Guo W, Cao Y, Huang D, Wang H, et al. Clinical and immunological features of severe and moderate coronavirus disease 2019. J Clin Invest. 2020;130:2620-9.

27. Zhu Z, Cai T, Fan L, Lou K, Hua X, Huang Z, et al. Clinical value of immune-inflammatory parameters to assess the severity of coronavirus disease 2019. Int J Infect Dis. 2020;95:332-9.

28. Guzik TJ, Mohiddin SA, Dimarco A, Patel V, Savvatis K, MarelliBerg FM, et al. COVID-19 and the cardiovascular system: implications for risk assessment, diagnosis, and treatment options. Cardiovasc Res. 2020;116:1666-87.

29. Huang C, Wang Y, Li X, Ren L, Zhao J, Hu Y, et al. Clinical features of patients infected with 2019 novel coronavirus in Wuhan, China. Lancet. 2020;395:497-506.

30. Zhou F, Yu T, Du R, Fan G, Liu Y, Liu Z, et al. Clinical course and risk factors for mortality of adult inpatients with COVID-19 in Wuhan, China: a retrospective cohort study. Lancet. 2020;395: 1054-62.

31. Le RQ, Li L, Yuan W, Shord SS, Nie L, Habtemariam BA, et al. FDA approval summary: tocilizumab for treatment of chimeric antigen receptor $\mathrm{T}$ cell-induced severe or life-threatening cytokine release syndrome. Oncologist. 2018;23:943-7.

32. Biran N, Ip A, Ahn J, Go RC, Wang S, Mathura S, et al. Tocilizumab among patients with COVID-19 in the intensive care unit: a multicentre observational study. Lancet Rheumatol. 2020;2: e603-12.

33. Stone JH, Frigault MJ, Serling-Boyd NJ, Fernandes AD, Harvey L, Foulkes AS, et al. Efficacy of tocilizumab in patients hospitalized with Covid-19. N Engl J Med. 2020; Available from:. https://doi. org/10.1056/NEJMoa2028836.

34. Huet T, Beaussier H, Voisin O, Jouveshomme S, Dauriat G, Lazareth I, et al. Anakinra for severe forms of COVID-19: a cohort study. Lancet Rheumatol. 2020;2:e393-400.

35. Cavalli G, De Luca G, Campochiaro C, Della-Torre E, Ripa M, Canetti D, et al. Interleukin-1 blockade with high-dose anakinra in patients with COVID-19, acute respiratory distress syndrome, and hyperinflammation: a retrospective cohort study. Lancet Rheumatol. 2020;2:e325-31.

36. RECOVERY Collaborative Group, Horby P, Lim WS, Emberson JR, Mafham M, Bell JL, et al. Dexamethasone in Hospitalized Patients with Covid-19 - Preliminary Report. N Engl J Med. Massachusetts Medical Society. 2020. https://doi.org/10.1056/ NEJMoa2021436.

37. Beigel JH, Tomashek KM, Dodd LE, Mehta AK, Zingman BS, Kalil AC, et al. Remdesivir for the treatment of Covid-19 - final report. N Engl J Med. 2020;383:1813-26.

38. Januzzi JL. Troponin and BNP Use in COVID-19. Am. Coll. Cardiol. Cardiology magazine. https://www.acc.org/latest-incardiology/articles/2020/03/18/15/25/troponin-and-bnp-use-incovid19. Published 3/18/2020. Accessed 12/8/2020.

39. Sandoval, Y; Jaffe AS. Key points about myocardial injury and cardiac troponin in COVID-19. Am. Coll. Cardiol. Cardiology magazine. https://www.acc.org/latest-in-cardiology/articles/2020/ 07/17/08/00/key-points-about-myocardial-injury-and-cardiactroponin-in-covid-19. Published 7/17/2020. Accessed 12/5/2020

Publisher's Note Springer Nature remains neutral with regard to jurisdictional claims in published maps and institutional affiliations. 